\title{
Stem cell transplantation for multiple myeloma
}

\author{
Shaji Kumar \\ Division of Hematology and Department of Internal Medicine, Mayo Clinic, Rochester, Minnesota, \\ USA
}

\section{Abstract}

Purpose of review-Autologous stem cell transplantation (SCT) has been an integral part of the management of multiple myeloma, especially for younger patients. During the past decade, several highly effective therapies have become available for treatment of myeloma, raising questions about the role of SCT for its management. This review focuses on recent findings in the context of previous studies demonstrating a benefit for SCT compared with conventional therapies.

Recent findings-Recent clinical trials have primarily focused on improving patient outcome by integrating the novel agents into various stages of myeloma therapy, including initial therapy resulting in improvement of disease control at the time of SCT, incorporation into conditioning regimens and finally, using maintenance approaches to prolong the response from SCT. In addition, recent trials also have demonstrated a potential role for tandem SCT in selected patients. However, the role of allogeneic SCT still remains to be better defined.

Summary-Patients with myeloma have more effective choices for therapy today and better outcome. Recent and ongoing trials will allow better selection of therapy, preferably in an individualized fashion, potentially translating into prolonged survival.

\section{Keywords}

allogeneic; autologous; multiple myeloma; stem cell transplantation

\section{Introduction}

Stem cell transplantation (SCT), mostly autologous, has been the treatment of choice for patients with multiple myeloma considered eligible for the procedure. Several randomized trials have demonstrated an advantage for SCT compared with conventional therapy alone and formed the basis for this approach [1,2]. During the past decade, several new agents have been introduced for treatment of multiple myeloma, with treatment efficacy rivaling those previously seen only in the context of SCT $[3,4,5 \bullet \bullet$. The results with these new agents, alone or in various combinations, have changed the treatment paradigm for patients with myeloma and have fueled an intense debate regarding the current role of SCT [6••]. This review discusses the role of SCT for management of myeloma in this new era of 'highly effective but non-curative' therapy. 


\section{Autologous stem cell transplantation in the prenovel agent era}

High-dose therapy (HDT) for management of multiple myeloma was introduced over two decades ago based on the ability of high doses of chemotherapy to overcome innate and acquired drug resistance. Introduction of the stem cell rescue allowed well tolerated administration of myeloablative doses of chemotherapy alone or in combination with radiation. The widespread acceptance of this treatment modality for management of myeloma was based on the favorable results from several randomized control trials [1,2,7]. Although the initial trials laid the foundation for autologous stem cell transplantation (ASCT) approaches for myeloma, subsequent trials have systematically refined the role of this approach. The initial French trials and the Medical Research Council VII trial demonstrated an improved response duration and overall survival (OS) for ASCT compared with conventional therapies used at that time, mostly alkylator and steroid-based combination regimens. The details of the trials comparing SCT with conventional therapy are elaborated in Table 1. However, not all the randomized trials addressing this question were able to demonstrate an OS advantage for ASCT, though disease-free interval was clearly superior with the ASCT in most of these studies [8-10]. Patient selection criteria and use of different conditioning regimens have been used to explain the different results from these clinical trials. However, these seemingly conflicting results have allowed us to better understand the role of ASCT in myeloma and highlighted the possibility that not all transplant-eligible patients may require an ASCT. This is highlighted by the results of the PETHEMA trial that included only patients responding to the initial therapy in a phase III trial comparing ASCT with conventional therapies [10]. This trial failed to show any superiority for the transplant approach, underscoring the possibility that some of these patients with an excellent response to nontransplant treatments may not require SCT as part of initial therapy. Subsequent trials such as the S9321 and MAG91 failed to demonstrate a benefit for SCT compared with conventional therapy, but allowed for patients to get SCT as subsequent therapy following failure of initial regimen, and in effect asked the question whether early or delayed SCT were comparable [8,9]. This question was specifically addressed by the MAG90 trial that compared early SCT with SCT used as first line salvage therapy and demonstrated equivalent OS for the two approaches [11]. However, use of early ASCT was associated with a longer time without therapy and symptoms, a good surrogate for improved quality of life.

Additional trials and retrospective studies further allowed us to define the selection of patients, conditioning therapy and posttransplant maintenance strategies. Another InterGroupe Francophone du Myélome (IFM) trial specifically examined the role of total body irradiation (TBI) as part of conditioning therapy prior to ASCT transplant and showed better outcome when high-dose melphalan was used alone (without TBI) and has led to this being the current standard [12]. Although the randomized trials included patients younger than 65 years, studies suggest similar benefits for older patients who are considered eligible to undergo the procedure $[13,14 \bullet]$. Renal insufficiency is common at diagnosis, and patients with compromised renal function also can benefit from ASCT, if they are otherwise considered eligible [15]. Unlike other malignancies, response to preceding chemotherapy is not a prerequisite for consideration of ASCT in myeloma. In fact patients refractory to initial therapy of their disease can derive comparable benefits from ASCT as those responding to the pre-ASCT regimen [16]. Given this scenario, ASCT has been considered the standard of care for patients with myeloma who are eligible. A population-based study from Sweden suggested improved survival for patients with myeloma in the recent years, which was attributed mostly to wider use of ASCT.

The traditional approach to ASCT before the introduction of novel agents had been initial control of the disease using combination regimens such as vincristine, doxorubicin and 
dexamethasone (VAD) or single agent dexamethasone. The type of pretransplant therapy did not appear to have much impact on the outcome of SCT and likely reflects the low rates of deep responses [very good partial response (VGPR) and complete responses] seen with these regimens [17•]. However, alkylating agents are avoided as part of initial therapy due to their impact on the ability to collect stem cells [18]. Following 4-6 months of therapy, patients underwent stem cell collection with granulocyte colony-stimulating factor alone or in combination with chemotherapy, followed by immediate transplantation or continuation of alkylator or steroid-based treatment regimens with the aim of delayed transplantation at relapse [19]. Purging of the collected stem cells has not been beneficial and is not considered standard. Post-ASCT maintenance therapy has not been considered standard, with clinical trials examining steroids or interferon not showing significant benefit. In a small, randomized clinical trial, 85 patients received either maintenance treatment with interferon- $\alpha$ until relapse or no further treatment following initial ASCT [20]. Even though initial results appeared to demonstrate longer event-free survival(EFS) and OS with maintenance therapy, the differences were no longer significant with longer follow-up. Other approaches for posttransplant maintenance have included immunotherapy strategies such as dendritic cell vaccines.

\section{Prognostic factors in the context of autologous stem cell transplantation}

Several clinical and laboratory features have been identified that can impact the outcome of ASCT. These have included disease stage, presence of renal insufficiency, high plasma cell proliferative rate, presence of circulating plasma cells, elevated $\beta-2$ microglobulin (B2M) and lactate dehydrogenase, disease progressing on prior therapy, preexistent diagnosis of monoclonal gammopathy of undetermined significance and abnormal cytogenetics among others [21-26]. The strongest predictors of outcome following SCT have been the presence of cytogenetic abnormalities [21]. Myeloma is a genetically heterogeneous disease with nearly half of the patients with a hypodiploid karyotype, usually harboring translocations involving the immunoglobulin heavy chain locus on chromosome 14 [27]. The remaining patients typically have a hyperdiploid karyotype. Nearly half of the patients, belonging to either of these groups, can also have abnormalities of chromosome 13. In addition to these primary abnormalities, plasma cells can acquire additional abnormalities involving the $p 53$ gene on chromosome 17p. Patients with t $(4 ; 14), \mathrm{t}(14 ; 16)$, del 13 on conventional cytogenetics and $17 \mathrm{p}$ abnormalities have response durations less than a year compared with a median response duration of 24 months seen in the randomized trials [21]. Application of more sophisticated technology such as gene expression profiling has allowed us to identify sets of genes that can predict different outcomes following SCT approaches, but these are not available for routine testing at this time.

\section{Role of second autologous stem cell transplantation}

A second ASCT can be considered for management of multiple myeloma, either as salvage therapy in patients relapsing after previous ASCT or as consolidation therapy soon after the first ASCT (tandem ASCT). Investigators at the University of Arkansas initially reported on the use of sequential ASCT in their Total Therapy I protocol, which consisted of a series of induction regimens and two cycles of HDT [28].

Several randomized trials have directly addressed the question of single versus double upfront transplants (Table 2). In the IFM94 trial, the EFS (20 versus 10\%) and the OS (42 versus 21\%) at 7 years posttransplant doubled with addition of the second ASCT, even though there was only minor improvement in the combined complete remission and VGPR rate with double transplant (50 versus $42 \%$ ) [29]. The benefit was mostly restricted to those not achieving a VGPR with the first ASCT. In the Bologna 96 trial, addition of a second 
HDT prolonged EFS by 12 months and time to progression by 17 months, with a projected OS at 6 years of $44 \%$ for single transplant and $63 \%$ for double transplant $(P=0.3)[30 \bullet \cdot$. As with IFM94, patients failing to achieve a complete or near complete remission after the first HDT obtained the maximum benefit. Similarly, the HOVON24 trial also showed prolonged EFS for double ASCT without any improvement in the OS, whereas the MAG95 trial did not have improvement in either EFS or OS with the double ASCT $[31,32]$.

Although these trials have looked at two planned transplants in a 'back to back' fashion, the role of a second transplant as salvage therapy after previous transplants has not been studied in a prospective fashion. However, enough evidence exists from retrospective studies to consider this therapy in patients with relapsed disease [33,34]. In a report of 172 patients relapsing after one ASCT 54 patients received a second ASCT and the rest received salvage chemotherapy. Although there was a trend towards improved OS with repeat ASCT, there was no benefit for those relapsing less than 18 months from the initial ASCT with median survival less than 6 months compared with 3 years for those with a longer response from first ASCT. A European Bone Marrow Transplantation Registry (EBMTR) analysis of 'planned' sequential transplants (presumed tandem) or 'unplanned' (presumed salvage) showed a median survival from ASCT of 60 months for the planned group versus 51 months for the rest $(P=.05)$ [35]. The authors concluded that in order to improve survival with tandem ASCT, it should be performed before relapse and within 6-12 months of first ASCT.

In another interesting trial, Abdelkefi et al. [36••] randomized 195 patients, with de-novo symptomatic myeloma, younger than 60 years to receive either tandem ASCT upfront or one ASCT followed by a maintenance therapy with thalidomide for 6 months, with the option of a second ASCT at disease progression. In both arms, the initial therapy was thalidomidedexamethasone. The 3-year progression-free survival (PFS) and OS were 57 and 65\%, respectively with tandem ASCT compared with a PFS as well as OS of $85 \%$ with thalidomide maintenance. This trial raised the possibility that additional consolidation therapy with a short course of novel agent may be able to substitute for a tandem approach.

\section{Impact of novel agents, and the current role of autologous stem cell transplantation for multiple myeloma}

The past decade has seen dramatic changes in our approach to treatment of myeloma, primarily as a result of introduction of thalidomide, its analogue, lenalidomide and the proteasome inhibitor, bortezomib. The new drugs have had the maximum impact in the initial treatment of myeloma. Thalidomide in combination with dexamethasone was first studied and led to high response rates and deeper responses in patients with previously untreated myeloma. Although the phase III trials showed improved PFS with thalidomide and dexamethasone compared with dexamethasone or VAD, there was no significant improvement in the OS [3,37]. Given the high response rates, thalidomide-dexamethasone soon replaced the traditional VAD or pulse dexamethasone regimens as initial therapy of myeloma. Lenalidomide in combination with dexamethasone in phase II and III studies have resulted in over $90 \%$ response rates, and especially with long-term treatment have resulted in complete and near complete remission rates of over 50\%, hitherto seen only in the context of ASCT-based approaches $[5 \bullet \cdot 38,39]$. What was more striking has been the significant reduction in the early mortality rates in patients with multiple myeloma, especially when lenalidomide was combined with lower (weekly) doses of dexamethasone compared with traditional pulse doses [5••]. Similarly, bortezomib has also been studied in the setting of newly diagnosed multiple myeloma with high response rates [40]. Bortezomib in combination with dexamethasone was superior to VAD in a randomized French trial in terms of response rates and depth of responses, allowing for significant reduction in the requirement of tandem transplantation [41]. One could argue that the biggest contribution of 
these novel drugs has been the reduction in the early mortality, an aspect of disease control that ASCT has not been able to address considering that most of these happened prior to the patient receiving ASCT. These findings have led to the adoption of lenalidomide or bortezomib-based initial therapy of multiple myeloma by most of the practitioners (Fig. 1). The new agents have allowed development of initial treatment strategies that are well tolerated, highly effective, decrease the initial mortality, allow rapid control of disease with potential reversal of complications such as renal insufficiency and finally, allow continued treatment if a decision is made to delay ASCT.

Although the focus of clinical investigation has been to define the role of these new drugs in untreated myeloma, some trials have examined ways to incorporate these agents into conditioning regimens. Another trial added bortezomib to melphalan as part of a conditioning regimen for ASCT for myeloma and has found this to be well tolerated, and the results warrant a study in randomized setting [42].

The availability of these new drugs also renewed the interest in maintenance strategies after ASCT. Several ongoing and partially reported trials are evaluating the role of thalidomide or lenalidomide as maintenance therapy after ASCT [43-45]. The IFM99-02 trial randomized patients 2 months after tandem ASCT to receive no maintenance, pamidronate or pamidronate and thalidomide [43]. The response rates were significantly higher for the thalidomide arm, and this translated into an improved EFS of 52\% compared with $36 \%$ with no maintenance and $37 \%$ with pamidronate alone. However, long-term results of this trial have failed to demonstrate a survival advantage. Ongoing trials are evaluating the role of bortezomib or lenalidomide as maintenance after single ASCT.

\section{Allogeneic stem cell transplantation for multiple myeloma}

There has been limited success with allogeneic stem cell transplantation (allo-SCT) for treatment of myeloma, largely a result of high treatment-related toxicity in this patient population. This along with the paucity of well designed randomized trials precludes development of firm recommendation regarding the optimal use of this modality and should primarily be considered in the context of clinical trials. There is little doubt that a graft versus myeloma effect exists and is obvious from the higher rate of molecular responses following allo-SCT compared with ASCT that in turn translates into longer remissions [46]. In a study using PCR-based assessment of clonal markers, half of those undergoing alloSCT achieved a molecular complete remission compared with $16 \%$ of ASCT and had a significantly lower relapse rate and longer PFS (35 versus 110 months) [47].

Most of the initial reports on the utility of allogeneic approaches have come from small studies or transplant registries (Table 3) [48-51]. The recent trials have attempted to compare the allogeneic approach with ASCT, with enrichment for patients with high-risk disease. In a retrospective case-matched analysis from the EBMTR, patients treated with allogeneic bone marrow transplantation (allo-BMT) were compared with a similar group of patients who received ASCT [49]. The OS was significantly better for the ASCT than for allo-BMT, with a median survival of 34 and 18 months, respectively. The poorer survival in allo-BMT patients could be attributed mostly to the higher transplantation-related mortality (TRM) (41 versus 13\%). Among patients surviving the first year, there was a trend for better OS and EFS for allo-BMT. Although the treatment-related toxicity has improved over time, it still remains high. Data from the EBMTR suggest that the TRM with allogeneic transplant fell from $46 \%$ at 2 years for transplants done during 1983-1993 to 30\% for those done during 1994-1998 [52].

Nonmyeloablative or reduced intensity conditioning (RIC) allo-SCT relies primarily on the immunological effects of the graft, with the conditioning chemotherapy primarily facilitating 
engraftment [53-55]. There have been two randomized trials that have attempted to compare ASCT with allo-SCT (Table 3). The IFM99-03/99-04 clinical trials studied patients with high-risk myeloma (B2M >3 mg/l and chromosome 13 deletion) [56]. Sixty-five patients with a human leukocyte antigen (HLA) identical sibling donor were assigned to receive RIC allogeneic SCT (IFM99-03 trial), and 219 patients without an HLA-identical sibling donor were assigned to undergo second ASCT (IFM99-04 protocol). The incidence of acute graft versus host disease (GVHD) was 32\%, chronic GVHD was $43 \%$ and TRM was $10 \%$.

Responses included $62.2 \%$ with complete remission, $20 \%$ with partial remission and $18 \%$ stable disease/ progressive disease at 2 months. On an intent-to-treat basis, the median OS and EFS did not differ significantly between the groups (35 and 25 months in the IFM99-03 trial versus 41 and 30 months in the IFM99-04 trial, respectively). When the 166 patients randomly assigned in the tandem ASCT protocol were compared with those undergoing allogeneic transplant, the EFS was similar (35 versus 31.7 months), with a trend for better OS with tandem ASCT (median, 47.2 versus 35 months; $P=0.07$ ). Thus, in spite of improved TRM, there was no advantage for allo-SCT. In the Italian trial, 108 patients less than 65 years with newly diagnosed multiple myeloma received standard ASCT followed by lowdose TBI conditioning and HLA-matched sibling peripheral blood SCT (median of 2-4 months from ASCT) or went on to receive a second ASCT [57 • ]. At a median follow-up of 3 years, TRM was $11 \%$ with allo-SCT versus $4 \%$ with double ASCT; complete remission rate was 46 versus $16 \%$; OS was 84 versus $62 \%$ and PFS was 75 versus $41 \%$, all significant differences.

\section{Current approach to the patient with newly diagnosed multiple myeloma}

The current approach to the management of newly diagnosed patients is described in Fig. 1.We recommend initial therapy with a lenalidomide or bortezomib-containing regimen for 2-4 cycles, followed by stem cell mobilization and harvest. Lenalidomide has been associated with reduced stem cell harvest, a feature that seems to be more common in the older patients and those receiving more than 4 cycles of treatment. On the basis of previous clinical trials demonstrating equivalent survival for early versus delayed ASCT, it is reasonable to continue with initial therapy to maximum benefit possible titrated to minimum toxicity and consider ASCT at the first relapse. Given the rather poor outcome following ASCT in patients with high-risk features (adverse cytogenetics, high-proliferation status and high B2M), our approach has been to maximize the benefit with the initial therapy incorporating agents such as bortezomib, which have been shown to overcome the impact of the poor prognostic factors, and to consider ASCT as a second line treatment. For patients failing to achieve a VGPR with their first ASCT, tandem ASCT or consolidation therapy with one of the new agents to the maximum response can be considered. Allogeneic approaches remain an option for younger patients with poor risk disease or for those with a rapid relapse after their first ASCT.

\section{Conclusion}

ASCT is an effective therapy for multiple myeloma and should be considered an option for all patients younger than 65 years and older patients with excellent functional status. The role of ASCT continues to evolve with the development of effective agents for multiple myeloma. The response rates seen with new drugs rival that of ASCT and raise important questions regarding the role and timing of ASCT. The time has come for us to reexamine many of the questions that were assumed to have been answered. Clearly, ASCT remains an effective therapy with known durability in terms of disease control, an aspect of the new drugs that still needs to be defined. Meanwhile, it is clear that the new drugs cannot obviate the need for ASCT in the majority of patients with multiple myeloma, considered transplant eligible. The new drugs can, however, be incorporated into the treatment algorithm along 
with ASCT to improve the outcome of patients. These drugs will be used at all stages of the disease management including initial therapy, as part of conditioning therapy and potentially as consolidation or maintenance therapy after ASCT. In addition, it offers patients the option of delaying ASCT to later in the course of the disease. Finally, the role of allo-SCT still remains to be defined, and some of the recently completed trials will allow us to enhance our understanding.

\section{References and recommended reading}

Papers of particular interest, published within the annual period of review, have been highlighted as:

- of special interest

•- of outstanding interest

Additional references related to this topic can also be found in the Current World Literature section in this issue (pp. 187-188).

1. Attal M, Harousseau JL, Stoppa AM, et al. Intergroupe Francais du Myelome. A prospective, randomized trial of autologous bone marrow transplantation and chemotherapy in multiple myeloma. N Engl J Med 1996;335:91-97. [PubMed: 8649495]

2. Child JA, Morgan GJ, Davies FE, et al. High-dose chemotherapy with hematopoietic stem-cell rescue for multiple myeloma. N Engl J Med 2003;348:1875-1883. [PubMed: 12736280]

3. Rajkumar SV, Blood E, Vesole DH, et al. Phase III clinical trial of thalidomide plus dexamethasone compared with dexamethasone alone in newly diagnosed multiple myeloma: a clinical trial coordinated by the Eastern Cooperative Oncology Group. J Clin Oncol 2006;24:431-436. [PubMed: 16365178]

4. Richardson PG, Sonneveld P, Schuster MW, et al. Bortezomib or high-dose dexamethasone for relapsed multiple myeloma. N Engl J Med 2005;352:2487-2498. [PubMed: 15958804]

5••. Rajkumar SV, Jacobus S, Callander N, et al. A randomized trial of lenalidomide plus high-dose dexamethasone $(\mathrm{RD})$ versus lenalidomide plus low-dose dexamethasone (Rd) in newly diagnosed multiple myeloma (E4A03): a trial coordinated by the Eastern Cooperative Oncology Group. Blood 2007;110:74. ASH Annual Meeting Abstracts. [PubMed: 17371947] . This trial brought about a paradigm change in the treatment of myeloma by demonstrating better outcome with lower doses of steroids despite a lowering of response rates. In addition, the 2-year survival was one of the highest seen with any drug therapy for myeloma.

6••. Kumar SK, Rajkumar SV, Dispenzieri A, et al. Improved survival in multiple myeloma and the impact of novel therapies. Blood 2008;111:2516-2520. [PubMed: 17975015] . This retrospective study, for the first time, showed that the outcome of patients has improved in the recent years, and this is likely related to the introduction of novel therapies.

7. Palumbo A, Bringhen S, Petrucci MT, et al. Intermediate-dose melphalan improves survival of myeloma patients aged 50 to 70: results of a randomized controlled trial. Blood 2004;104:30523057. [PubMed: 15265788]

8. Fermand JP, Katsahian S, Divine M, et al. High-dose therapy and autologous blood stem-cell transplantation compared with conventional treatment in myeloma patients aged 55 to 65 years: long-term results of a randomized control trial from the Group Myelome-Autogreffe. J Clin Oncol 2005;23:9227-9233. [PubMed: 16275936]

9. Barlogie B, Kyle RA, Anderson KC, et al. Standard chemotherapy compared with high-dose chemoradiotherapy for multiple myeloma: final results of phase III US Intergroup Trial S9321. J Clin Oncol 2006;24:929-936. [PubMed: 16432076]

10. Blade J, Rosinol L, Sureda A, et al. High-dose therapy intensification compared with continued standard chemotherapy in multiple myeloma patients responding to the initial chemotherapy: longterm results from a prospective randomized trial from the Spanish cooperative group PETHEMA. Blood 2005;106:3755-3759. [PubMed: 16105975] 
11. Fermand JP, Ravaud P, Chevret S, et al. High-dose therapy and autologous peripheral blood stem cell transplantation in multiple myeloma: up-front or rescue treatment? Results of a multicenter sequential randomized clinical trial. Blood 1998;92:3131-3136. [PubMed: 9787148]

12. Moreau P, Facon T, Attal M, et al. Comparison of $200 \mathrm{mg} / \mathrm{m}^{2}$ melphalan and $8 \mathrm{~Gy}$ total body irradiation plus $140 \mathrm{mg} / \mathrm{m}^{2}$ melphalan as conditioning regimens for peripheral blood stem cell transplantation in patients with newly diagnosed multiple myeloma: final analysis of the Intergroupe Francophone du Myélome 9502 randomized trial. Blood 2002;99:731-735. [PubMed: 11806971]

13. Siegel DS, Desikan KR, Mehta J, et al. Age is not a prognostic variable with autotransplants for multiple myeloma. Blood 1999;93:51-54. [PubMed: 9864145]

14. Kumar SK, Dingli D, Lacy MQ, et al. Autologous stem cell transplantation in patients of 70 years and older with multiple myeloma: results from a matched pair analysis. Am J Hematol 2008;83:614-617. [PubMed: 18429054] . In a case-control study, older patients with myeloma (>70 years) are shown to have equivalent benefit from ASCT as their younger peers.

15. Gertz MA, Lacy MQ, Dispenzieri A, et al. Impact of age and serum creatinine value on outcome after autologous blood stem cell transplantation for patients with multiple myeloma. Bone Marrow Transplant 2007;39:605-611. [PubMed: 17369867]

16. Kumar S, Lacy MQ, Dispenzieri A, et al. High-dose therapy and autologous stem cell transplantation for multiple myeloma poorly responsive to initial therapy. Bone Marrow Transplant 2004;34:161-167. [PubMed: 15133489]

17•. Kumar SK, Dingli D, Dispenzieri A, et al. Impact of pretransplant therapy in patients with newly diagnosed myeloma undergoing autologous SCT. Bone Marrow Transplant 2008;41:1013-1019. [PubMed: 18332915] . This study demonstrates that for patients reaching ASCT, the type of initial therapy does not have significant impact on their long-term outcome.

18. Knudsen LM, Rasmussen T, Jensen L, Johnsen HE. Reduced bone marrow stem cell pool and progenitor mobilisation in multiple myeloma after melphalan treatment. Med Oncol 1999;16:245254. [PubMed: 10618687]

19. Alegre A, Tomas JF, Martinez-Chamorro C, et al. Comparison of peripheral blood progenitor cell mobilization in patients with multiple myeloma: high-dose cyclophosphamide plus GM-CSF vs GCSF alone. Bone Marrow Transplant 1997;20:211-217. [PubMed: 9257889]

20. Cunningham D, Powles R, Malpas J, et al. A randomized trial of maintenance interferon following high-dose chemotherapy in multiple myeloma: long-term follow-up results. Br J Haematol 1998;102:495-502. [PubMed: 9695964]

21. Gertz MA, Lacy MQ, Dispenzieri A, et al. Clinical implications of $t(11 ; 14)(q 13 ; q 32), t(4 ; 14)$ (p16.3;q32), and -17p13 in myeloma patients treated with high-dose therapy. Blood 2005;106:2837-2840. [PubMed: 15976175]

22. Gutierrez NC, Castellanos MV, Martin ML, et al. Prognostic and biological implications of genetic abnormalities in multiple myeloma undergoing autologous stem cell transplantation: $t(4 ; 14)$ is the most relevant adverse prognostic factor, whereas RB deletion as a unique abnormality is not associated with adverse prognosis. Leukemia 2007;21:143-150. [PubMed: 17024116]

23. O'Shea D, Giles C, Terpos E, et al. Predictive factors for survival in myeloma patients who undergo autologous stem cell transplantation: a single-centre experience in 211 patients. Bone Marrow Transplant 2006;37:731-737. [PubMed: 16501593]

24. Avet-Loiseau H, Attal M, Moreau P, et al. Genetic abnormalities and survival in multiple myeloma: the experience of the Intergroupe Francophone du Myelome. Blood 2007;109:3489_ 3495. [PubMed: 17209057]

25. Rajkumar SV, Fonseca R, Dispenzieri A, et al. Effect of complete response on outcome following autologous stem cell transplantation for myeloma. Bone Marrow Transplant 2000;26:979-983. [PubMed: 11100277]

26. Rajkumar SV, Fonseca R, Lacy MQ, et al. Beta 2-microglobulin and bone marrow plasma cell involvement predict complete responders among patients undergoing blood cell transplantation for myeloma. Bone Marrow Transplant 1999;23:1261-1266. [PubMed: 10414913]

27. Fonseca R, Blood E, Rue M, et al. Clinical and biologic implications of recurrent genomic aberrations in myeloma. Blood 2003;101:4569-4575. [PubMed: 12576322] 
28. Barlogie B, Jagannath S, Vesole DH, et al. Superiority of tandem autologous transplantation over standard therapy for previously untreated multiple myeloma. Blood 1997;89:789-793. [PubMed: 9028309]

29. Attal M, Harousseau JL, Facon T, et al. Single versus double autologous stem-cell transplantation for multiple myeloma. N Engl J Med 2003;349:2495-2502. [PubMed: 14695409]

30••. Cavo M, Tosi P, Zamagni E, et al. Prospective, randomized study of single compared with double autologous stem-cell transplantation for multiple myeloma: Bologna 96 clinical study. $\mathbf{J}$ Clin Oncol 2007;25:2434-2441. [PubMed: 17485707] . This randomized control trial compared single with double autologous SCT and demonstrates improved progression-free survival without any impact on the overall survival.

31. Sonneveld $\mathrm{P}$, van der Holt B, Vellenga E, et al. Intensive versus double intensive therapy in untreated multiple myeloma: final analysis of the HOVON 24 trial. Blood 2005;106:2545. ASH Annual Meeting Abstracts.

32. Fermand JP, Alberti C, Marolleau JP. Single versus tandem high dose therapy (HDT) supported with autologous blood stem cell (ABSC) transplantation using unselected or CD34-enriched ABSC: results of a two by two designed randomized trial in 230 young patients with multiple myeloma (MM). Hematol J 2003;4(Suppl 1):S59.

33. Tricot G, Jagannath S, Vesole DH, et al. Relapse of multiple myeloma after autologous transplantation: survival after salvage therapy. Bone Marrow Transplant 1995;16:7-11. [PubMed: 7581132]

34. Elice F, Raimondi R, Tosetto A, et al. Prolonged overall survival with second on-demand autologous transplant in multiple myeloma. Am J Hematol 2006;81:426-431. [PubMed: 16680735]

35. Morris C, Iacobelli S, Brand R, et al. Benefit and timing of second transplantations in multiple myeloma: clinical findings and methodological limitations in a European Group for Blood and Marrow Transplantation registry study. J Clin Oncol 2004;22:1674-1681. [PubMed: 15037597]

36••. Abdelkefi A, Ladeb S, Torjman L, et al. Single autologous stem-cell transplantation followed by maintenance therapy with thalidomide is superior to double autologous transplantation in multiple myeloma: results of a multicenter randomized clinical trial. Blood 2008;111:1805-1810. [PubMed: 17875806] . A randomized control trial showing that a second (tandem) ASCT can be replaced by defined duration of therapy with one of the novel agents.

37. Cavo M, Zamagni E, Tosi P, et al. Superiority of thalidomide and dexamethasone over vincristinedoxorubicindexamethasone (VAD) as primary therapy in preparation for autologous transplantation for multiple myeloma. Blood 2005;106:35-39. [PubMed: 15761019]

38. Lacy M, Gertz M, Dispenzieri A, et al. Lenalidomide plus dexamethasone (rev/dex) in newly diagnosed myeloma: response to therapy, time to progression, and survival. Blood 2006;108:798. abstract.

39. Zonder JA, Crowley J, Hussein MA, et al. Superiority of lenalidomide (len) plus high-dose dexamethasone (HD) compared to HD alone as treatment of newly-diagnosed multiple myeloma (NDMM): results of the randomized, double-blinded, placebo-controlled SWOG trial S0232. Blood 2007;110:77. ASH Annual Meeting Abstracts.

40. Jagannath S, Durie B, Wolf JL, et al. A phase 2 study of bortezomib as first-line therapy in patients with multiple myeloma. Blood 2004;104:A333. abstract.

41. Harousseau JL, Mathiot C, Attal M, et al. VELCADE/Dexamethasone (Vel/D) versus VAD as induction treatment prior to autologous stem cell transplantion (ASCT) in newly diagnosed multiple myeloma (MM): updated results of the IFM 2005/01 trial. Blood 2007;110:450. ASH Annual Meeting Abstracts. [PubMed: 17371943]

42. Hollmig K, Stover J, Talamo G, et al. Addition of bortezomib (VelcadeTM) to high dose melphalan (Vel-Mel) as an effective conditioning regimen with autologous stem cell support in multiple myeloma (MM). Blood 2004;104:929. ASH Annual Meeting Abstracts.

43. Attal M, Harousseau JL, Leyvraz S, et al. Maintenance therapy with thalidomide improves survival in patients with multiple myeloma. Blood 2006;108:3289-3294. [PubMed: 16873668] 
44. Stewart AK, Chen CI, Howson-Jan K, et al. Results of a multicenter randomized phase II trial of thalidomide and prednisone maintenance therapy for multiple myeloma after autologous stem cell transplant. Clin Cancer Res 2004;10:8170-8176. [PubMed: 15623591]

45. Sahebi F, Spielberger R, Kogut NM, et al. Maintenance thalidomide following single cycle autologous peripheral blood stem cell transplant in patients with multiple myeloma. Bone Marrow Transplant 2006;37:825-829. [PubMed: 16565743]

46. Tricot G, Vesole DH, Jagannath S, et al. Graft-versus-myeloma effect: proof of principle. Blood 1996;87:1196-1198. [PubMed: 8562947]

47. Martinelli G, Terragna C, Zamagni E, et al. Molecular remission after allogeneic or autologous transplantation of hematopoietic stem cells for multiple myeloma. J Clin Oncol 2000;18:22732281. [PubMed: 10829048]

48. Arora M, McGlave PB, Burns LJ, et al. Results of autologous and allogeneic hematopoietic cell transplant therapy for multiple myeloma. Bone Marrow Transplant 2005;35:1133-1140. [PubMed: 15834435]

49. Bjorkstrand BB, Ljungman P, Svensson H, et al. Allogeneic bone marrow transplantation versus autologous stem cell transplantation in multiple myeloma: a retrospective case-matched study from the European Group for Blood and Marrow Transplantation. Blood 1996;88:4711-4718. [PubMed: 8977265]

50. Alyea E, Weller E, Schlossman R, et al. Outcome after autologous and allogeneic stem cell transplantation for patients with multiple myeloma: impact of graft-versus-myeloma effect. Bone Marrow Transplant 2003;32:1145-1151. [PubMed: 14647268]

51. Kuruvilla J, Shepherd JD, Sutherland HJ, et al. Long-term outcome of myeloablative allogeneic stem cell transplantation for multiple myeloma. Biology of blood and marrow transplantation 2007;13:925-931. [PubMed: 17640596]

52. Gahrton G, Svensson H, Cavo M, et al. Progress in allogenic bone marrow and peripheral blood stem cell transplantation for multiple myeloma: a comparison between transplants performed 1983-93 and 1994-8 at European Group for Blood and Marrow Transplantation centres. Br J Haematol 2001;113:209-216. [PubMed: 11360893]

53. Crawley C, Lalancette M, Szydlo R, et al. Outcomes for reduced-intensity allogeneic transplantation for multiple myeloma: an analysis of prognostic factors from the Chronic Leukaemia Working Party of the EBMT. Blood 2005;105:4532-4539. [PubMed: 15731182]

54. Maloney DG, Molina AJ, Sahebi F, et al. Allografting with nonmyeloablative conditioning following cytoreductive autografts for the treatment of patients with multiple myeloma. Blood 2003;102:3447-3454. [PubMed: 12855572]

55. Kroger N, Schwerdtfeger R, Kiehl M, et al. Autologous stem cell transplantation followed by a dose-reduced allograft induces high complete remission rate in multiple myeloma. Blood 2002;100:755-760. [PubMed: 12130482]

56. Garban F, Attal M, Michallet M, et al. Prospective comparison of autologous stem cell transplantation followed by dose-reduced allograft (IFM99-03 trial) with tandem autologous stem cell transplantation (IFM99-04 trial) in high-risk de novo multiple myeloma. Blood 2006;107:3474-3480. [PubMed: 16397129]

57••. Bruno B, Rotta M, Patriarca F, et al. A comparison of allografting with autografting for newly diagnosed myeloma. N Engl J Med 2007;356:1110-1120. [PubMed: 17360989] . The first randomized trial to show any benefit for alloSCT over ASCT in myeloma. 


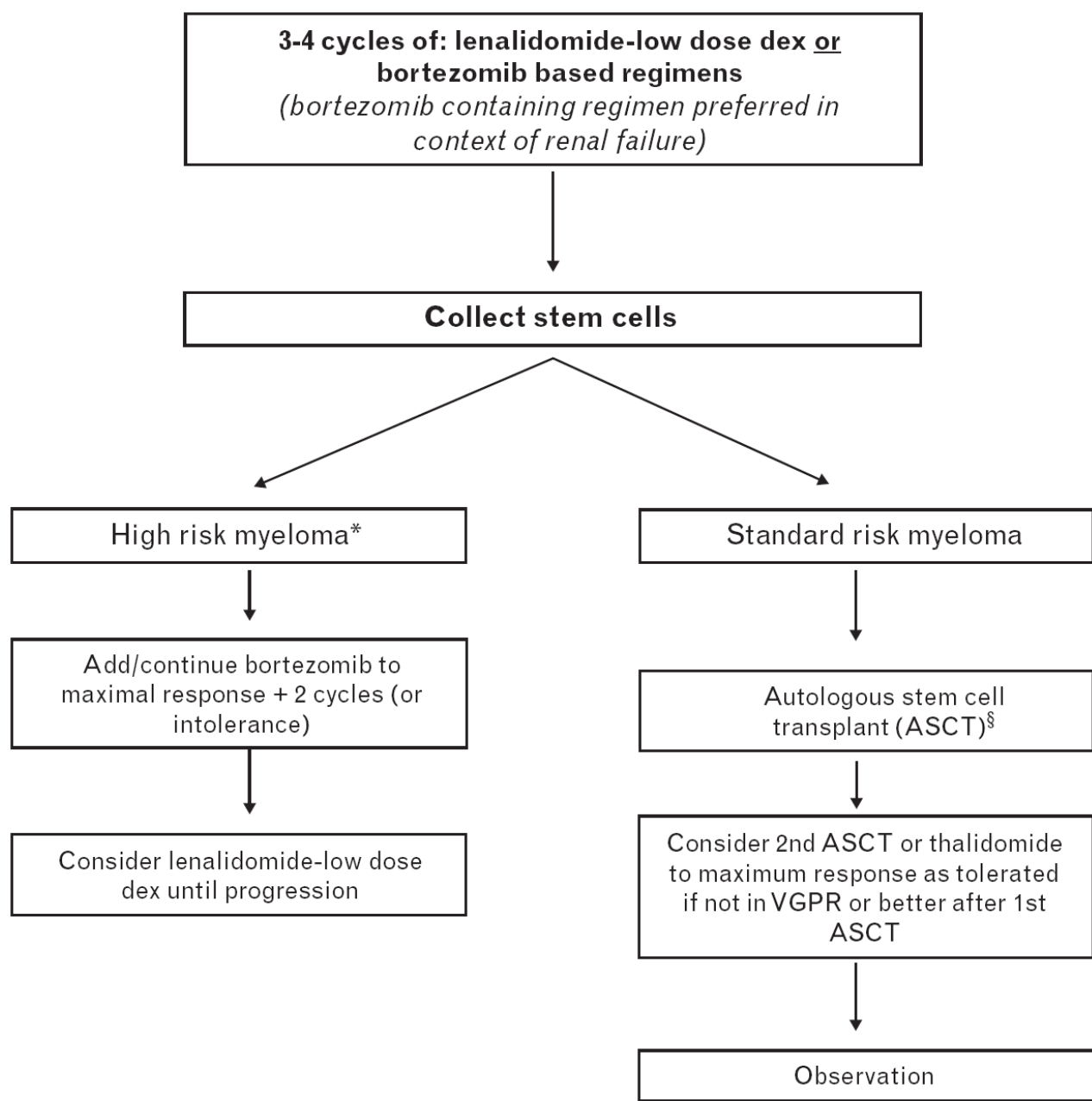

*High risk myeloma:

Del 17p, t( $4 ; 14), \mathrm{t}(14 ; 16)$ by FISH

Deletion 13 or hypodiploidy on conventional cytogenetics

Plasma cell labeling index $\geq 3 \%$

Figure 1. Decision tree for treatment approach in patients with newly diagnosed myeloma considered eligible for autologous stem cell transplantation

ASCT, autologous stem cell transplantation; FISH, fluorescence in-situ hybridization;

VGPR, very good partial response. 


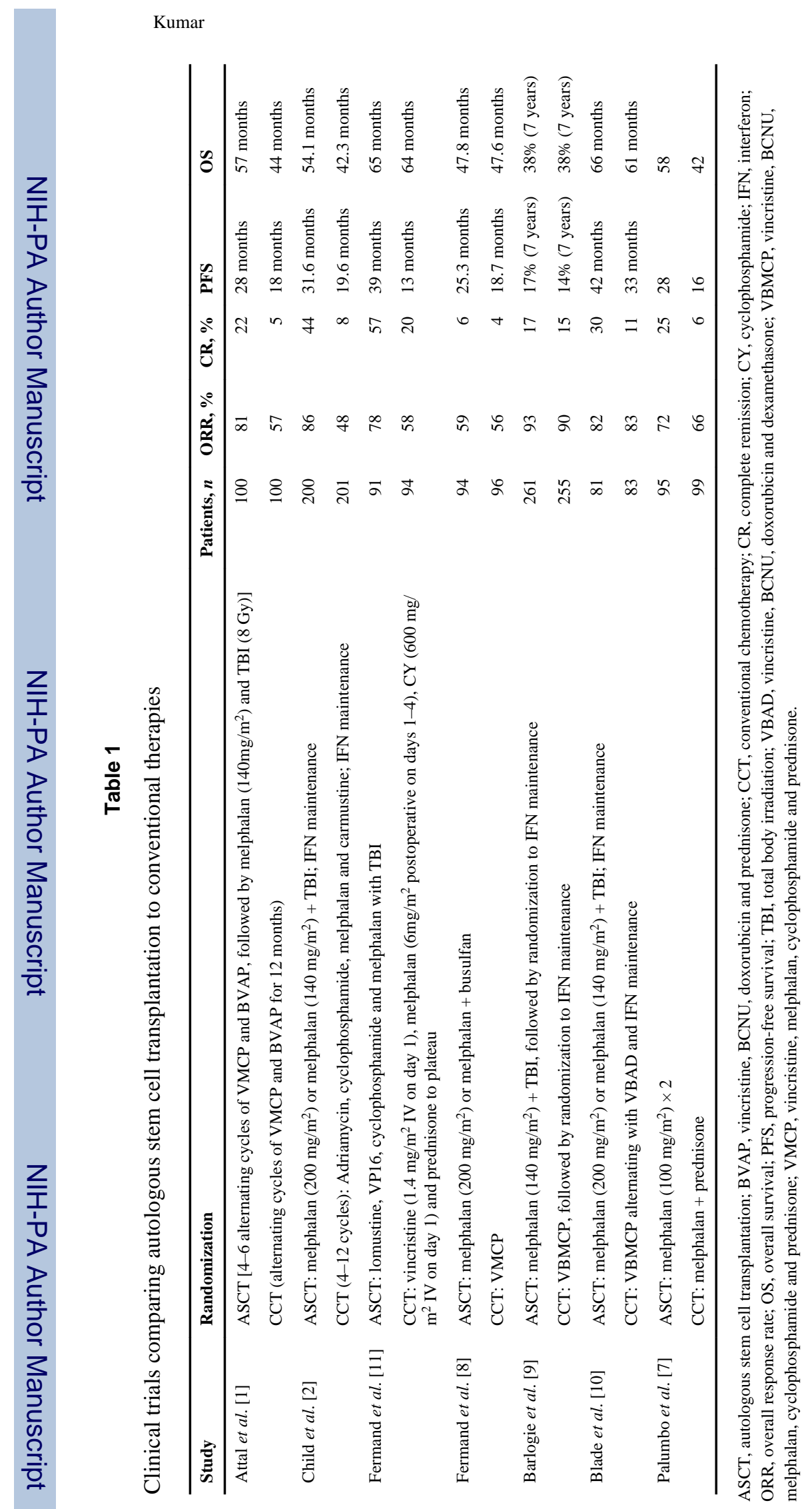

Curr Opin Oncol. Author manuscript; available in PMC 2010 July 21. 


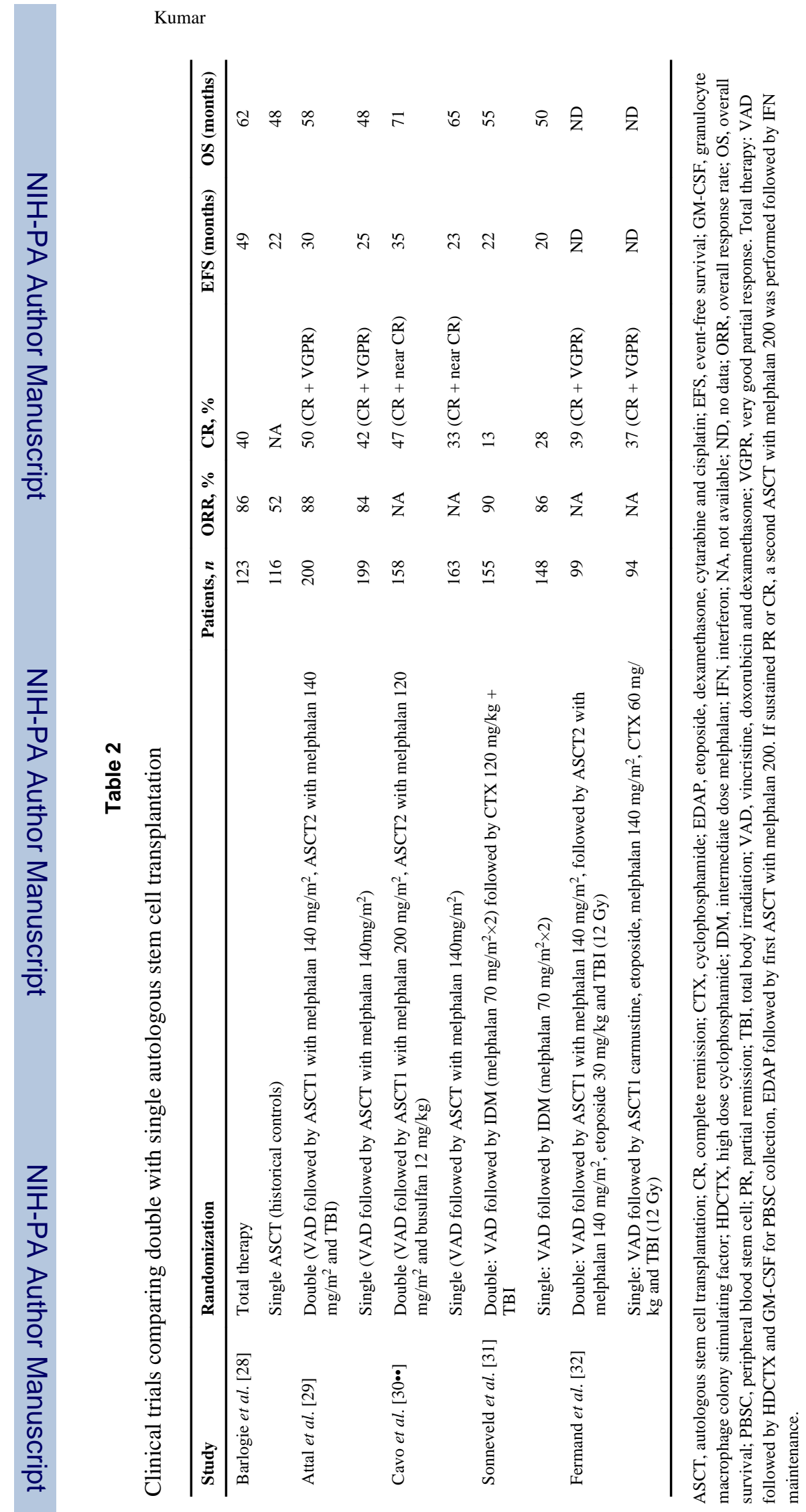

Curr Opin Oncol. Author manuscript; available in PMC 2010 July 21. 


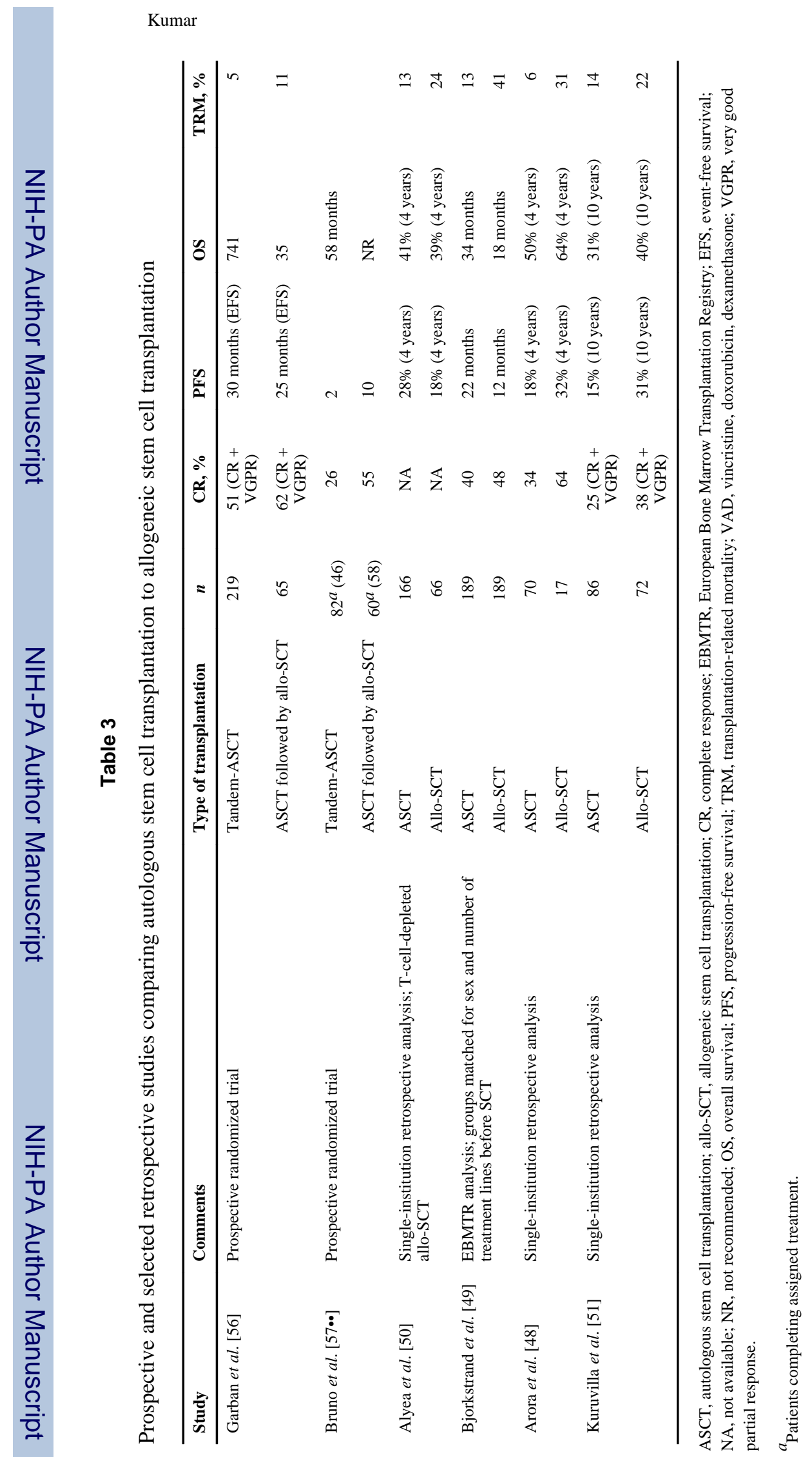

Curr Opin Oncol. Author manuscript; available in PMC 2010 July 21. 